\title{
Theoretical evidence for low charging overpotentials of superoxide discharge products in metal-oxygen batteries
}

Byungju Lee ${ }^{1,2}$, Gabin Yoon ${ }^{1,2}$, Hee-Dae Lim ${ }^{1}$, In-Suk Choi ${ }^{3}$ and Kisuk Kang ${ }^{2, *}$

1 Department of Materials Science and Engineering, Research Institute of Advanced Materials (RIAM), Seoul National University, 1 Gwanak-ro, Gwanak-gu, Seoul, 151-742, Republic of Korea

2 Center for Nanoparticles Research, Institute for Basic Science (IBS), Seoul National University, 1 Gwanak-ro, Gwanak-gu, Seoul, 151-742, Republic of Korea

3 High Temperature Energy Materials Research Center, Korea Institute of Science and Technology, Seoul, 136-791, Republic of Korea

Supporting information 


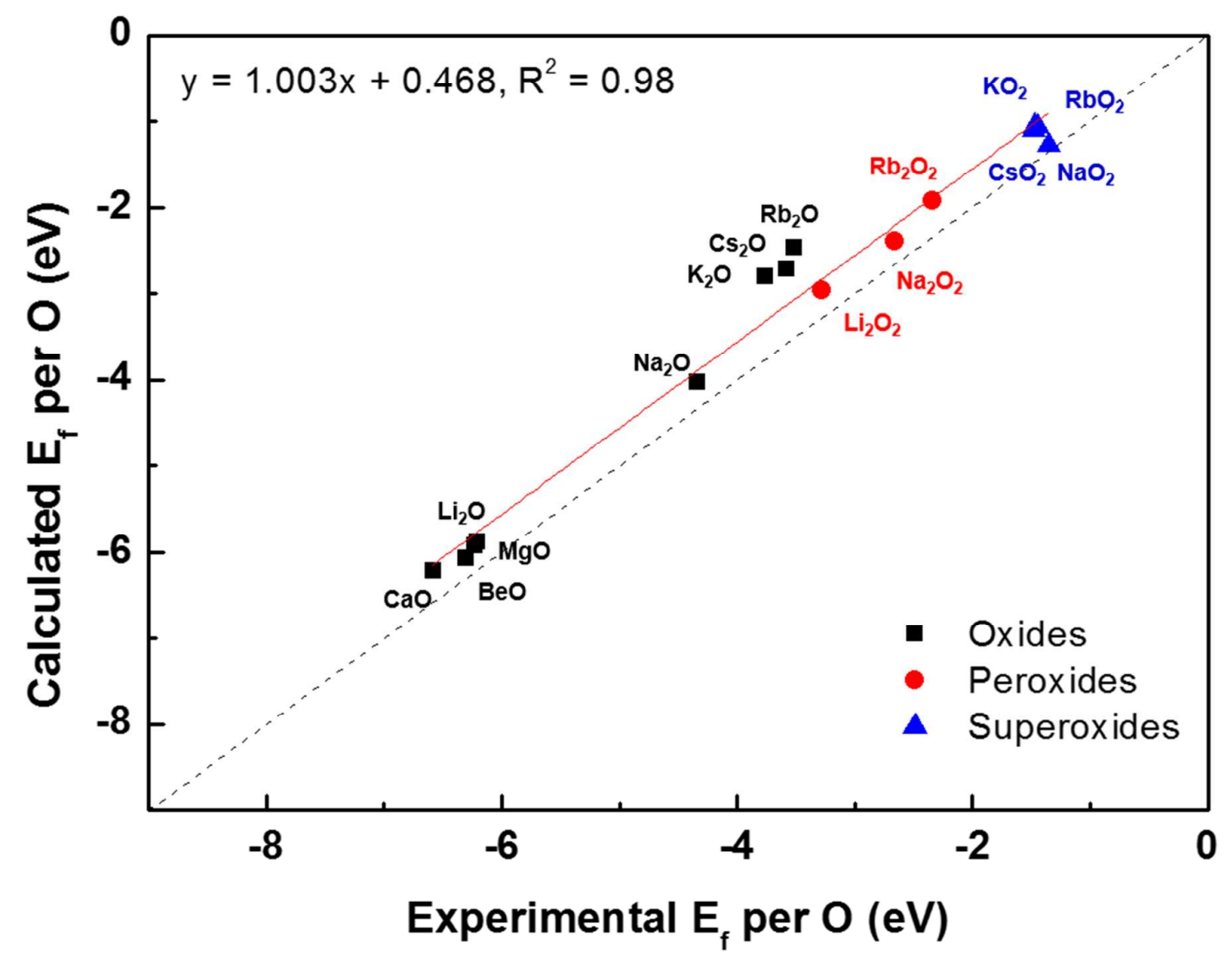

Figure S1. Formation energy per oxygen atom calculated in HSE $(\alpha=0.48)$ at $0 \mathrm{~K}$ versus the formation enthalpy measured at standard state, $298 \mathrm{~K}$ and 1 atm. Black squares, red circles, blue triangles indicate oxides, peroxides, superoxides respectively. Black dash line shows exact correspondence between experiment and calculation, while red solid line is linear regression fitted to all data points which yields slope of 1.003 and y-intercept of 0.468 . Note that separate fittings for oxides, peroxides, superoxides in the same data set result in overbinding correction of $0.590,0.335$, and $0.317 \mathrm{eV} / \mathrm{O}$ respectively. However, relative conductivity differences among the discharge products in this study were not largely changed (Table S1). 


\begin{tabular}{lcccc}
\hline & \multicolumn{2}{c}{ Electronic conductivity $(\mathbf{S} / \mathbf{c m})$} & \multicolumn{2}{c}{ Ionic conductivity $\mathbf{( S / c m )}$} \\
\hline & $\begin{array}{c}\text { Single overbinding } \\
\text { correction }\end{array}$ & $\begin{array}{c}\text { Separate overbinding } \\
\text { correction }\end{array}$ & $\begin{array}{c}\text { Single overbinding } \\
\text { correction }\end{array}$ & $\begin{array}{c}\text { Separate overbinding } \\
\text { correction }\end{array}$ \\
$\mathrm{LiO}_{2}$ & $2 \times 10^{-17}$ & $1 \times 10^{-19}$ & $6 \times 10^{-17}$ & $3 \times 10^{-19}$ \\
$\mathrm{NaO}_{2}$ & $4 \times 10^{-17}$ & $2 \times 10^{-19}$ & $3 \times 10^{-9}$ & $2 \times 10^{-11}$ \\
$\mathrm{Li}_{2} \mathrm{O}_{2}$ & $3 \times 10^{-21}$ & $3 \times 10^{-22}$ & $1 \times 10^{-19}$ & $1 \times 10^{-20}$ \\
$\mathrm{Na}_{2} \mathrm{O}_{2}$ & $2 \times 10^{-19}$ & $2 \times 10^{-20}$ & $3 \times 10^{-20}$ & $3 \times 10^{-21}$ \\
\hline
\end{tabular}

Table S1. Calculated conductivity of discharge products using single overbinding correction energy and separately applied overbinding correction energy. 


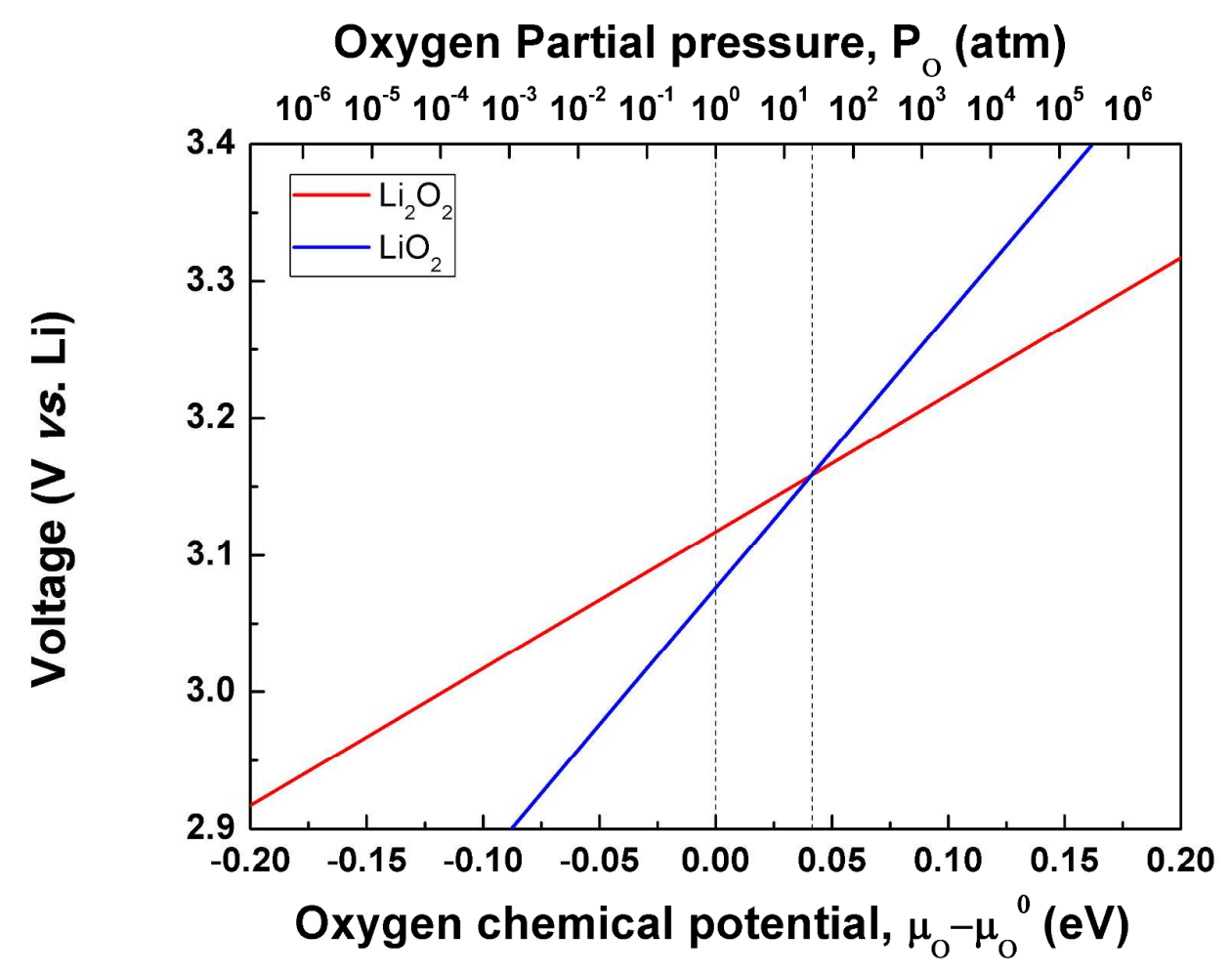

Figure S2. Relationship between cell voltage and oxygen partial pressure, in the $\mathrm{Li} / \mathrm{O}_{2}$ cell. Higher cell voltage is related with more negative Gibbs free energy, according to Nernst equation; $\mu_{L i}-\mu_{L i}{ }^{0}=-n F E_{\text {cell }}$, where $\mu_{L i}$ is chemical potential of metal Li in structure, $\mu_{L i}{ }^{0}$ is chemical potential of pure metal, $\mathrm{n}$ is the number of charge of metal ion, $\mathrm{F}$ is faraday constant, $E_{\text {cell }}$ is the voltage of the cell. HSE functional was used for energy calculation. $\mathrm{LiO}_{2}$ becomes more stable than $\mathrm{Li}_{2} \mathrm{O}_{2}$ in about 25 atm of oxygen partial pressure. 
$\gamma=\frac{1}{2 A}\left[G_{\text {slab }}-G_{\text {bulk }}\right]=\frac{1}{2 A}\left[G_{\text {slab }}-N_{O} \mu_{O}^{\text {bulk }}-N_{M} \mu_{M}^{\text {bulk }}\right]$

(a)

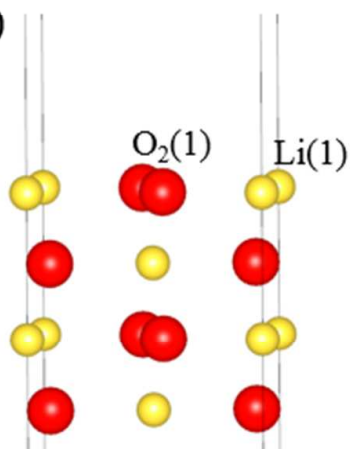

(001)

(d)

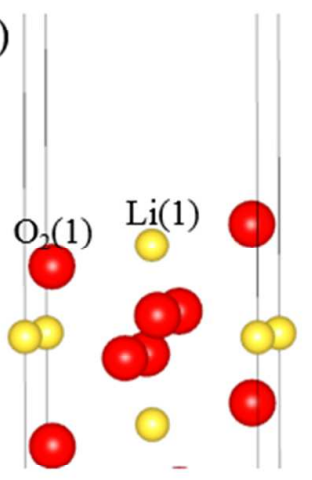

(100)

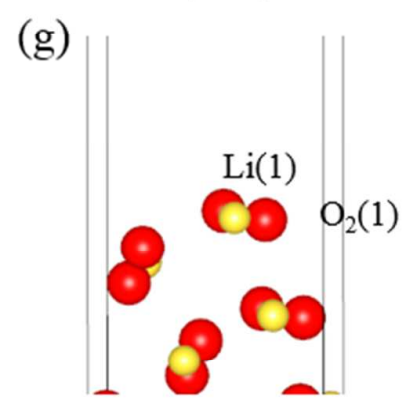

(111) (b)

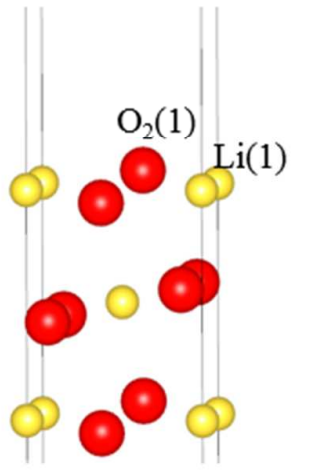

(010)

(e)

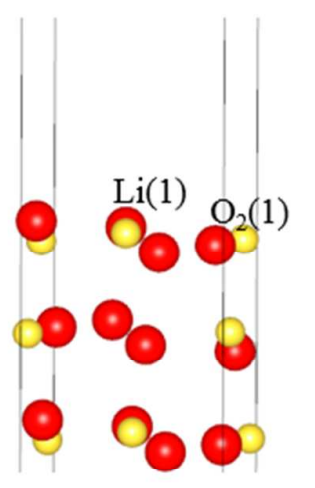

(101)

(h)

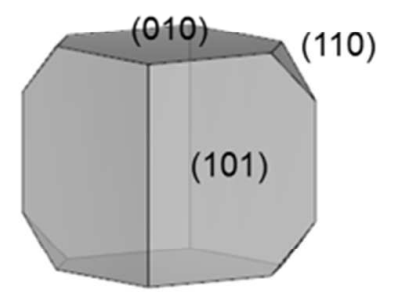

(c)

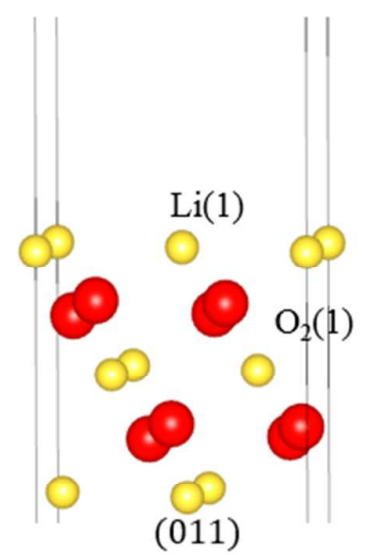

(f)

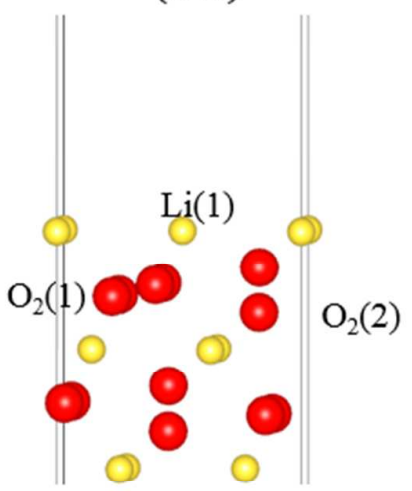

(110)

Figure S2. (a-g) The low index surface structures of $\mathrm{LiO}_{2}$ and their terminations. (h) Wulff shape of $\mathrm{LiO}_{2}$ was constructed from calculated surface energies. For surface energy calculations, a slab/vacuum geometry composed of repeating slabs and vacuum layers was adopted. 


\begin{tabular}{|c|c|c|c|}
\hline Orientation & Termination & $\mathbf{L i}_{2} \mathbf{O}_{2}$ limit & $\mathrm{O}_{2}$ limit \\
\hline \multirow{3}{*}{$(001)$} & $\mathrm{O}_{2}(1)-\mathrm{Li}(1)^{*}$ & 31 & 31 \\
\hline & $\mathrm{O}_{2}(1)$ & 47 & 53 \\
\hline & $\operatorname{Li}(1)$ & 71 & 64 \\
\hline \multirow{3}{*}{$(010)$} & $\mathrm{O}_{2}(1)-\mathrm{Li}(1)^{*}$ & 22 & 22 \\
\hline & $\mathrm{O}_{2}(1)$ & 24 & 35 \\
\hline & $\operatorname{Li}(1)$ & 221 & 210 \\
\hline \multirow{2}{*}{$(011)$} & $\mathrm{O}_{2}(1)-\mathrm{Li}(1)$ & 57 & 51 \\
\hline & $\mathrm{O}_{2}(1)$ & 45 & 51 \\
\hline \multirow{3}{*}{$(100)$} & $\mathrm{O}_{2}(1)-\mathrm{Li}(1)^{*}$ & 36 & 36 \\
\hline & $\mathrm{O}_{2}(1)$ & 29 & 37 \\
\hline & $\operatorname{Li}(1)$ & 58 & 50 \\
\hline \multirow{3}{*}{ (101) } & $\mathrm{O}_{2}(1)-\mathrm{Li}(1)^{*}$ & 18 & 18 \\
\hline & $\mathrm{O}_{2}(1)$ & 17 & 28 \\
\hline & $\operatorname{Li}(1)$ & 152 & 142 \\
\hline \multirow{4}{*}{ (110) } & $\mathrm{Li}(1)-\mathrm{O}_{2}(1)-\mathrm{O}_{2}(2)$ & 39 & 32 \\
\hline & $\mathrm{O}_{2}(1)-\mathrm{O}_{2}(2)$ & 38 & 45 \\
\hline & $\mathrm{O}_{2}(1)^{*}$ & 34 & 34 \\
\hline & $\mathrm{O}_{2}(2)^{*}$ & 29 & 29 \\
\hline \multirow{3}{*}{ (111) } & $\mathrm{O}_{2}(1)-\mathrm{Li}(1)^{*}$ & 25 & 25 \\
\hline & $\mathrm{O}_{2}(1)$ & 27 & 32 \\
\hline & $\operatorname{Li}(1)$ & 63 & 58 \\
\hline
\end{tabular}

Table S3. Calculated surface energies of low index surfaces. All possible terminations were considered. The spin-polarized generalized gradient approximation (GGA) type calculation was conducted using density functional theory (DFT). Perdew-Burke-Ernzerhof exchangecorrelation parameterization was used. The overbinding energy of oxygen corrected from reaction $\mathrm{Li}_{2} \mathrm{O}+1 / 2 \mathrm{O}_{2} \rightarrow \mathrm{Li}_{2} \mathrm{O}_{2}$. The surface free energy was calculated from energy difference between bulk phase and surface structure, $\gamma=\frac{1}{2 A}\left[G_{\text {slab }}-G_{b u l k}\right]=\frac{1}{2 A}\left[G_{\text {slab }}-\right.$ $\left.N_{O} \mu_{O}{ }^{\text {bulk }}-N_{M} \mu_{M}^{\text {bulk }}\right]$ where $\mathrm{G}_{\text {slab }} / \mathrm{G}_{\text {bulk }}, \mathrm{N}_{\mathrm{M}} / \mathrm{N}_{\mathrm{O}}$ and $\mu_{\mathrm{M}}^{\text {bulk }} / \mu_{\mathrm{O}}$ bulk indicate Gibbs free energy of surface structure/bulk phase, the number of metal/oxygen atom and chemical potential of metal and oxygen in bulk phase. Both maximum and minimum value of chemical potential, which were derived from M-O phase diagram, were considered for surface calculation of each phase. All possible terminations of low index surfaces were investigated to construct Wulff shape. A convergence test of the vacuum and slab thicknesses indicated that a vacuum thickness of $10 \AA$ and slab thickness $>20 \AA$ were sufficient for convergence within $1 \mathrm{meV} / \AA^{2}$ for the surface energies. More detailed computational condition and formulation can be found in ref. 20 of manuscript. 


$$
\Delta G=\frac{1}{2}\left[E_{\text {step }}-E_{\text {initial }}+\Delta N_{O} \cdot \mu_{o}^{0}+\Delta N_{L i} \cdot\left(\mu_{L i}{ }^{0}-e U\right)\right]
$$

(a)
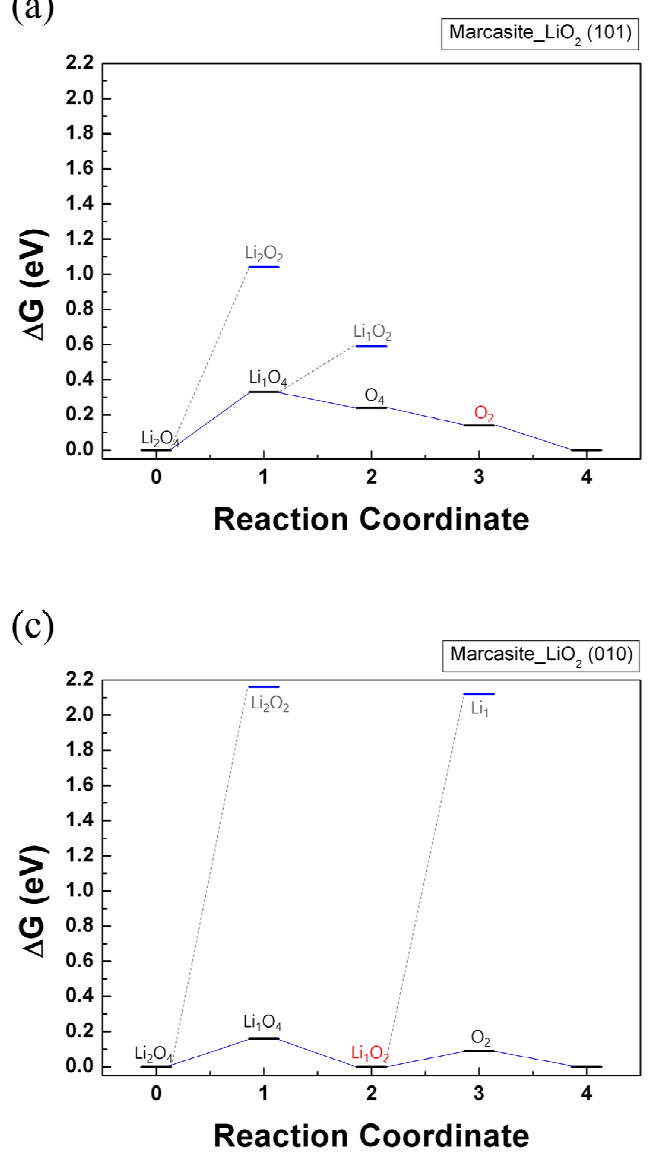

(b)

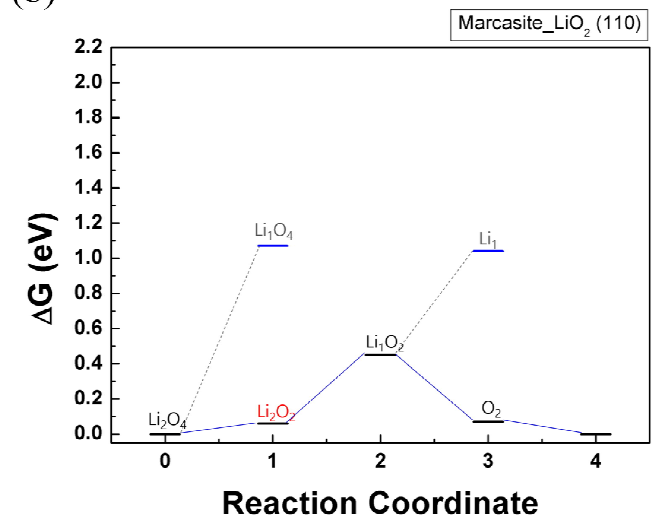

Figure S4. OER energy profile of $\mathrm{LiO}_{2}$ under the calculated equilibrium potential $\mathrm{U}=2.76 \mathrm{~V}$, in (a) (101) surface, (b) (110) surface and (c) (111) surface. During the OER, oxygen or metal atoms leave the surface upon charging. In the situation, two sequences are possible; the metal ion extraction which is the electrochemical reaction (black) or the oxygen molecule evolution, the chemical reaction (red). The energies of the intermediate steps of the OER were calculated by removing metal ions or oxygen molecules from the surface. One OER cycle can be modeled by considering the atoms in the surface of the unit cell. 

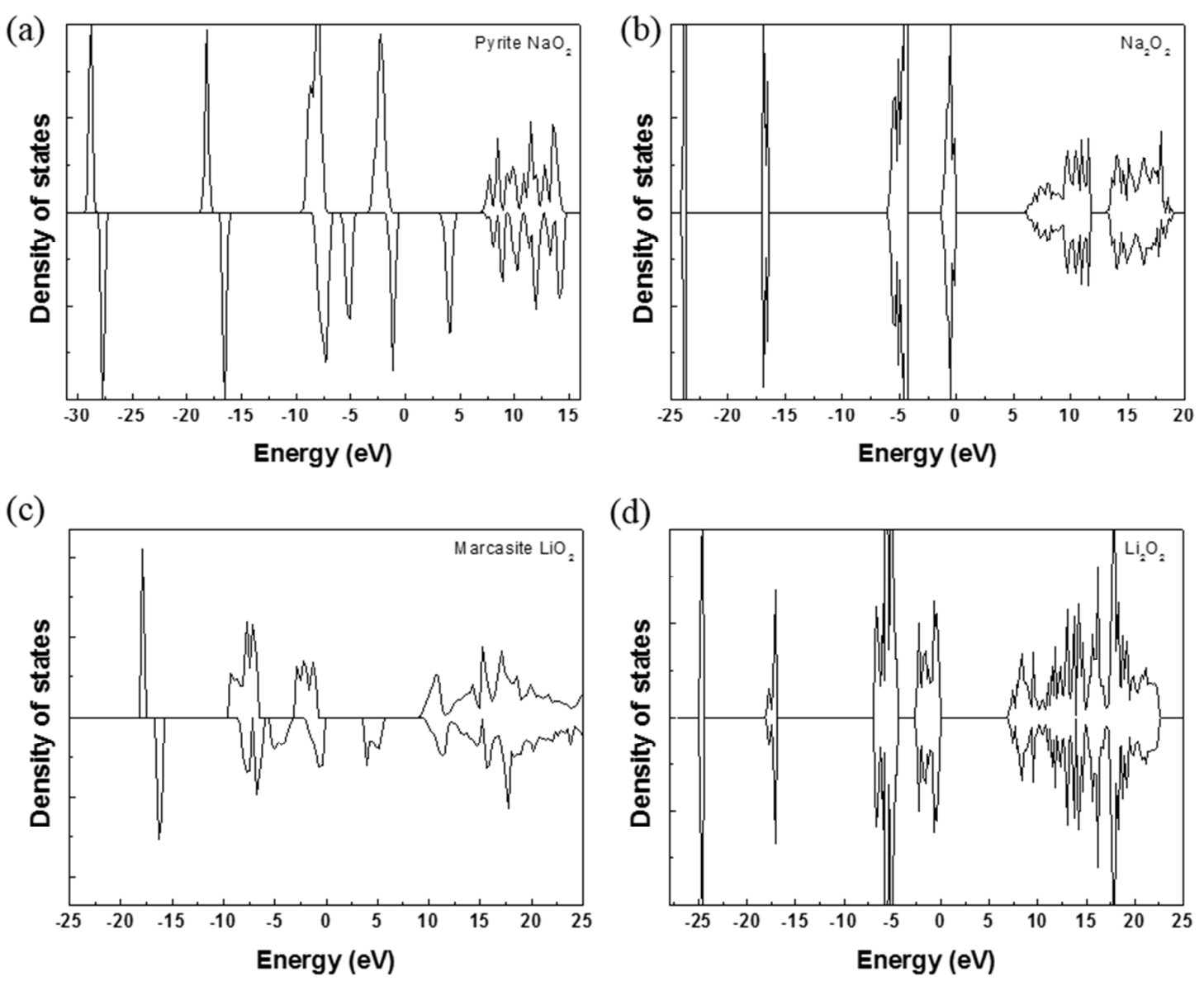

Figure S5. Density of state (DOS) of intrinsic (a) $\mathrm{NaO}_{2}$, (b) $\mathrm{Na}_{2} \mathrm{O}_{2}$, (c) $\mathrm{LiO}_{2}$ and (d) $\mathrm{Li}_{2} \mathrm{O}_{2}$, calculated with HSE hybrid functional. Mixing coefficient $(\alpha)$ was chosen as 0.48 . Calculated bandgap was 3.3, 5.8, 3.7 and $6.8 \mathrm{eV}$ for $\mathrm{NaO}_{2}, \mathrm{Na}_{2} \mathrm{O}_{2}, \mathrm{LiO}_{2}$ and $\mathrm{Li}_{2} \mathrm{O}_{2}$ respectively. 
(a)

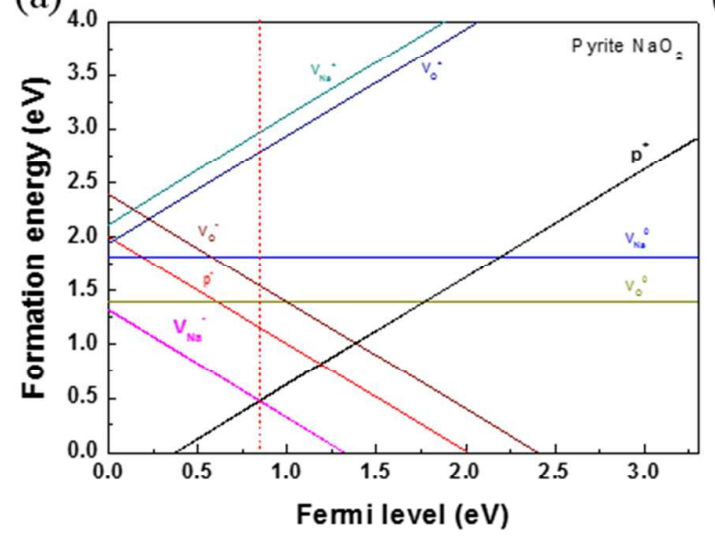

(c)

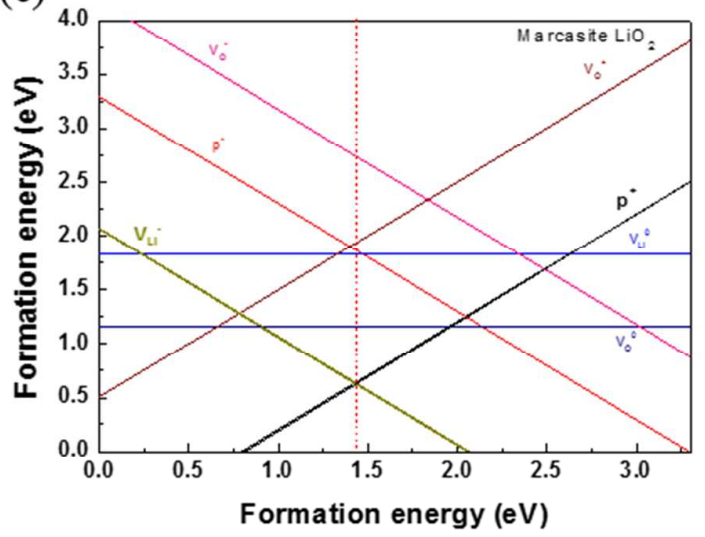

(b)

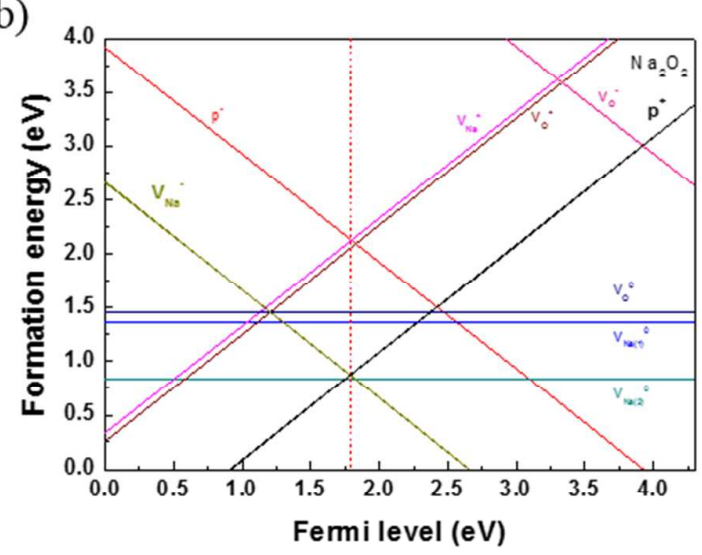

(d)

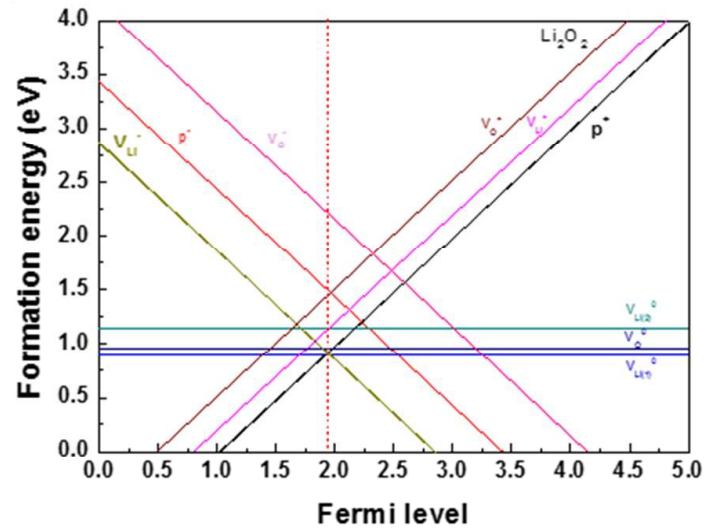

Figure S6. Formation energies of low energy defects in (a) $\mathrm{NaO}_{2}$, (b) $\mathrm{Na}_{2} \mathrm{O}_{2}$, (c) $\mathrm{LiO}_{2}$ and (d) $\mathrm{Li}_{2} \mathrm{O}_{2}$ versus Fermi energy. Red dash line is Fermi energy which satisfies charge neutrality. 


\begin{tabular}{|c|c|c|c|}
\hline $\mathrm{NaO}_{2}$ & $\begin{array}{l}\text { Concentration } \\
\left(\text { sites } / \mathbf{c m}^{-3}\right)\end{array}$ & $\mathrm{Na}_{2} \mathrm{O}_{2}$ & $\begin{array}{l}\text { Concentration } \\
\left(\text { sites } / \mathbf{c m}^{-3}\right)\end{array}$ \\
\hline $\mathbf{p}^{+}$ & 2.2.E+16 & $\mathbf{p}^{+}$ & $2.3 E+08$ \\
\hline $\mathrm{p}^{-}$ & 1.7.E+01 & $\mathrm{p}^{-}$ & 7.0E-15 \\
\hline $\mathrm{V}(\mathrm{Na})^{0}$ & 3.2.E-05 & $\mathrm{V}(\mathrm{Na})^{0}$ & $3.4 \mathrm{E}+09$ \\
\hline $\mathrm{V}(\mathrm{Na})^{+}$ & 1.1.E-22 & $\mathrm{V}(\mathrm{Na})^{+}$ & 3. $4 \mathrm{E}-12$ \\
\hline $\mathrm{V}(\mathrm{Na})^{-}$ & 2.2.E+16 & $\mathrm{V}(\mathrm{Na})^{-}$ & $2.3 E+08$ \\
\hline $\mathrm{V}(0)^{0}$ & 2.2.E-01 & $\mathrm{V}(\mathrm{O})^{0}$ & $1.8 \mathrm{E}-02$ \\
\hline $\mathrm{V}(\mathrm{O})^{+}$ & 4.7.E-23 & $\mathrm{V}(\mathrm{O})^{+}$ & $6.9 \mathrm{E}-12$ \\
\hline $\mathrm{V}(\mathrm{O})^{-}$ & 9.4.E-06 & $\mathrm{V}(\mathrm{O})^{-}$ & $5.6 \mathrm{E}-65$ \\
\hline $\mathrm{V}(\mathrm{Na})^{2+}$ & 2.8.E-33 & $\mathrm{V}(\mathrm{Na})^{2+}$ & $7.4 \mathrm{E}-33$ \\
\hline $\mathrm{V}(\mathrm{Na})^{2-}$ & 1.6.E-21 & $\mathrm{V}(\mathrm{Na})^{2-}$ & $8.0 \mathrm{E}-71$ \\
\hline $\mathrm{V}(\mathrm{O})^{2+}$ & 9.5.E-26 & $\mathrm{V}(\mathrm{O})^{2+}$ & $2.8 \mathrm{E}-39$ \\
\hline $\mathrm{V}(\mathrm{O})^{2-}$ & 4.7.E-22 & $\mathrm{V}(\mathrm{O})^{2-}$ & $4.0 \mathrm{E}-126$ \\
\hline $\mathrm{Na}_{i}{ }^{0}$ & 5.2.E-17 & $\mathrm{Na}_{\mathrm{i}}{ }^{0}$ & $2.8 \mathrm{E}-39$ \\
\hline $\mathrm{Na}^{+}$ & $3.5 . E+04$ & $\mathrm{Na}_{i}^{+}$ & $1.8 \mathrm{E}-44$ \\
\hline $\mathrm{Na}_{i}^{-}$ & 2.7.E-32 & $\mathrm{Na}_{\mathrm{i}}$ & $1.0 \mathrm{E}-72$ \\
\hline $\mathrm{V}\left(\mathrm{O}_{2}\right)^{0}$ & 1.3.E-34 & $\mathrm{V}\left(\mathrm{O}_{2}\right)^{0}$ & $1.3 \mathrm{E}-86$ \\
\hline $\mathrm{V}\left(\mathrm{O}_{2}\right)^{+}$ & 3.5.E-01 & $\mathrm{V}\left(\mathrm{O}_{2}\right)^{+}$ & $1.0 \mathrm{E}-58$ \\
\hline $\mathrm{V}\left(\mathrm{O}_{2}\right)^{-}$ & 5.3.E-62 & $\mathrm{V}\left(\mathrm{O}_{2}\right)^{-}$ & $2.3 \mathrm{E}-118$ \\
\hline
\end{tabular}

Table S3. Concentration of various defects in $\mathrm{NaO}_{2}$ and $\mathrm{Na}_{2} \mathrm{O}_{2}$ at Fermi energy, which is taken to the point which satisfies charge neutrality. 


\begin{tabular}{|c|c|c|c|}
\hline $\mathrm{LiO}_{2}$ & $\begin{array}{l}\text { Concentration } \\
\left(\text { sites } / \mathrm{cm}^{-3}\right)\end{array}$ & $\mathbf{L} \mathbf{i}_{2} \mathbf{O}_{2}$ & $\begin{array}{l}\text { Concentration } \\
\left(\text { sites } / \mathrm{cm}^{-3}\right)\end{array}$ \\
\hline $\mathbf{p}^{+}$ & $4.6 \mathrm{E}+11$ & $\mathbf{p}^{+}$ & $1.6 \mathbf{E}+07$ \\
\hline $\mathrm{p}^{-}$ & $6.8 \mathrm{E}-10$ & $\mathrm{p}^{-}$ & $3.2 \mathrm{E}+03$ \\
\hline $\mathrm{V}(\mathrm{Li})^{0}$ & $3.2 \mathrm{E}-09$ & $\mathrm{~V}(\mathrm{Li})^{0}$ & $3.4 \mathrm{E}+07$ \\
\hline $\mathrm{V}(\mathrm{Li})^{+}$ & $1.8 \mathrm{E}+22$ & $\mathrm{~V}(\mathrm{Li})^{+}$ & $2.8 \mathrm{E}-03$ \\
\hline $\mathbf{V}(\mathbf{L i})^{-}$ & $4.6 \mathrm{E}+11$ & $\mathbf{V}(\mathbf{L i})^{-}$ & $1.6 \mathbf{E}+07$ \\
\hline $\mathrm{V}(\mathrm{O})^{0}$ & $1.1 \mathrm{E}+03$ & $\mathrm{~V}(\mathrm{O})^{0}$ & $4.6 \mathrm{E}+06$ \\
\hline $\mathrm{V}(\mathrm{O})^{+}$ & $1.3 \mathrm{E}-10$ & $\mathrm{~V}(\mathrm{O})^{+}$ & $2.7 \mathrm{E}+02$ \\
\hline $\mathrm{V}(\mathrm{O})^{-}$ & $3.3 \mathrm{E}-24$ & $\mathrm{~V}(\mathrm{O})^{-}$ & $3.1 \mathrm{E}-15$ \\
\hline $\mathrm{V}(\mathrm{Li})^{2+}$ & - & $\mathrm{V}(\mathrm{Li})^{2+}$ & $4.8 \mathrm{E}-16$ \\
\hline $\mathrm{V}(\mathrm{Li})^{2-}$ & - & $\mathrm{V}(\mathrm{Li})^{2-}$ & $5.0 \mathrm{E}-35$ \\
\hline $\mathrm{V}(\mathrm{O})^{2+}$ & - & $\mathrm{V}(\mathrm{O})^{2+}$ & $1.8 \mathrm{E}-22$ \\
\hline $\mathrm{V}(\mathrm{O})^{2-}$ & $3.7 \mathrm{E}-18$ & $\mathrm{~V}(\mathrm{O})^{2-}$ & $5.6 \mathrm{E}-46$ \\
\hline $\mathrm{Li}_{1}{ }^{0}$ & $1.5 \mathrm{E}+00$ & $\mathrm{Li}_{i}{ }^{0}$ & $5.7 \mathrm{E}-28$ \\
\hline $\mathrm{Li}_{i}^{+}$ & $1.5 \mathrm{E}+02$ & $\mathrm{Li}_{i}^{+}$ & $2.1 \mathrm{E}-41$ \\
\hline $\mathrm{Li}_{\mathrm{i}}^{-}$ & $4.5 \mathrm{E}-02$ & $\mathrm{Li}_{i}^{-}$ & $2.0 \mathrm{E}-43$ \\
\hline $\mathrm{V}\left(\mathrm{O}_{2}\right)^{0}$ & $2.6 \mathrm{E}-20$ & $\mathrm{~V}\left(\mathrm{O}_{2}\right)^{0}$ & $4.0 \mathrm{E}-90$ \\
\hline $\mathrm{V}\left(\mathrm{O}_{2}\right)^{+}$ & $4.2 \mathrm{E}+06$ & $\mathrm{~V}\left(\mathrm{O}_{2}\right)^{+}$ & $2.4 \mathrm{E}-96$ \\
\hline $\mathrm{V}\left(\mathrm{O}_{2}\right)^{-}$ & $2.5 \mathrm{E}-22$ & $\mathrm{~V}\left(\mathrm{O}_{2}\right)^{-}$ & $7.8 \mathrm{E}-158$ \\
\hline
\end{tabular}

Table S4. Concentration of various defects in $\mathrm{LiO}_{2}$ and $\mathrm{Li}_{2} \mathrm{O}_{2}$ at Fermi energy, which is taken to the point which satisfies charge neutrality. 
(a)

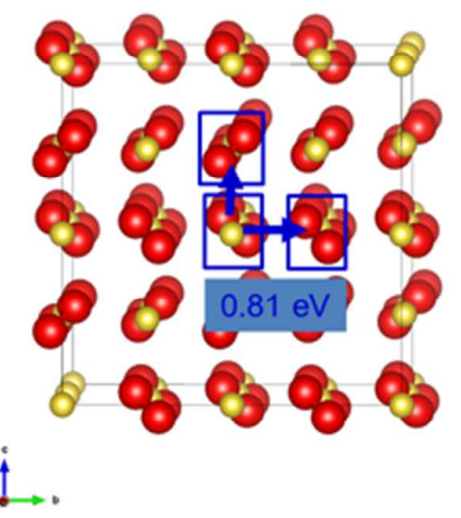

(c)

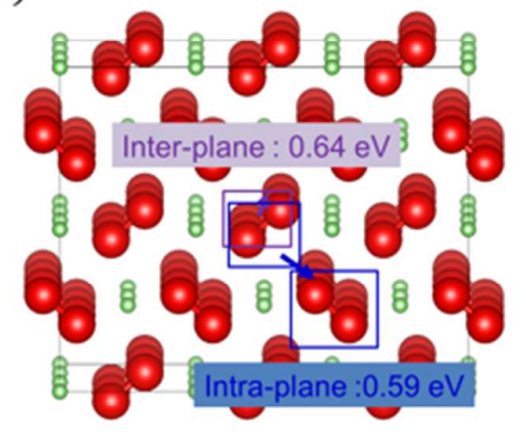

(b)

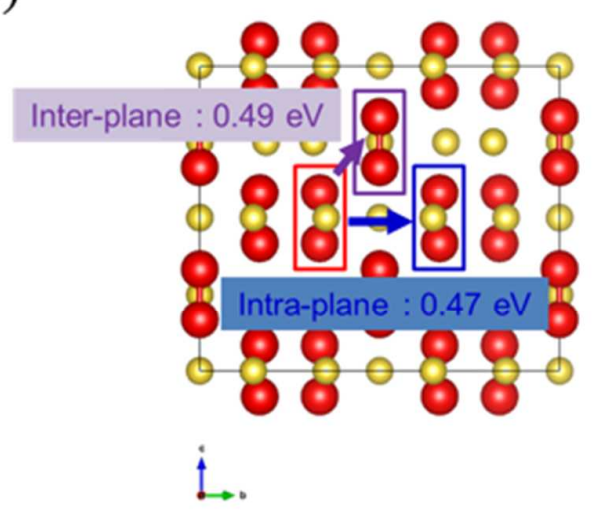

(d)

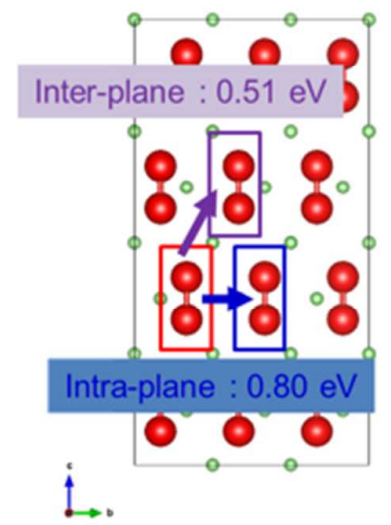

Figure S7. The hopping paths of hole and their hopping barrier in (a) $\mathrm{NaO}_{2}$, (b) $\mathrm{Na}_{2} \mathrm{O}_{2}$, (c) $\mathrm{LiO}_{2}$ and (d) $\mathrm{Li}_{2} \mathrm{O}_{2}$, calculated with HSE hybrid functional. 
(a)

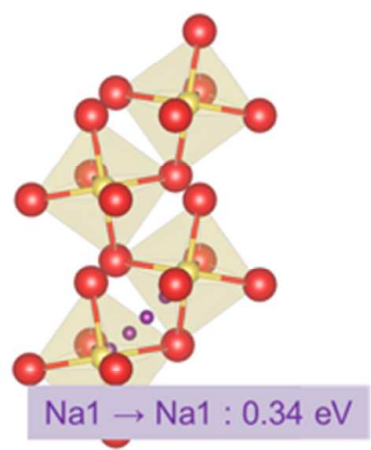

(c)

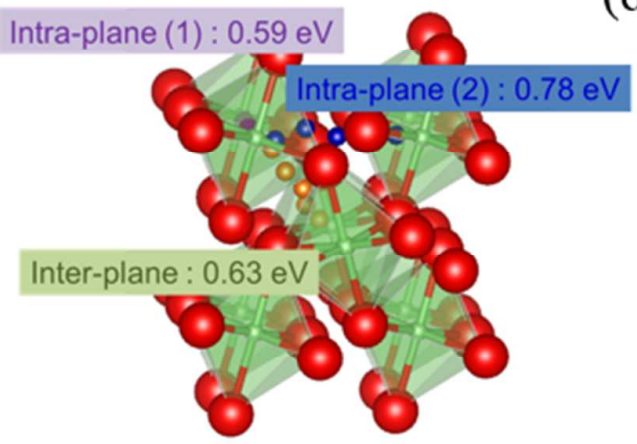

(b)

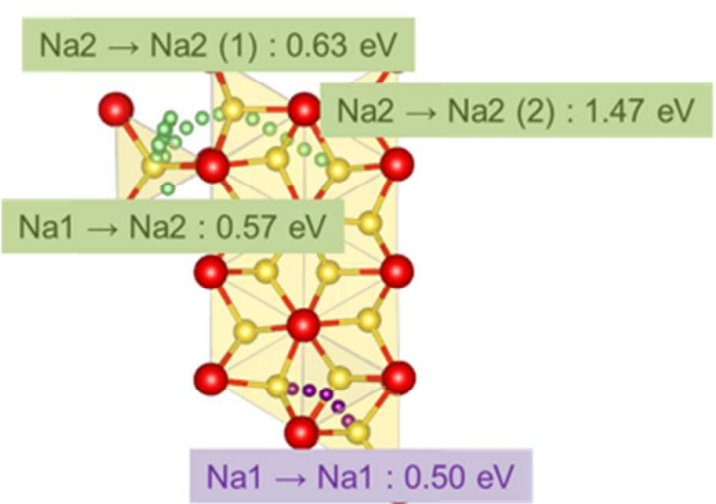

(d)

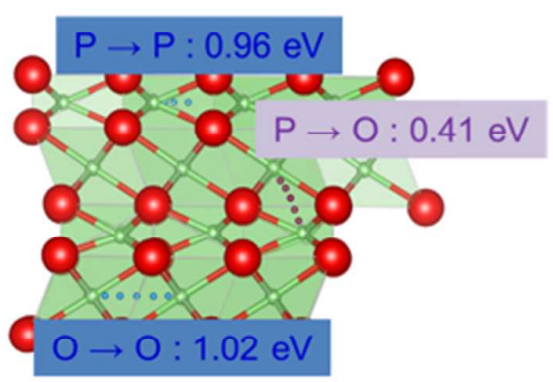

Figure S8. The hopping paths of negatively charged metal vacancy and their hopping barrier in (a) $\mathrm{NaO}_{2}$, (b) $\mathrm{Na}_{2} \mathrm{O}_{2}$, (c) $\mathrm{LiO}_{2}$ and (c) $\mathrm{Li}_{2} \mathrm{O}_{2}$, calculated with GGA functional and NEB method. 


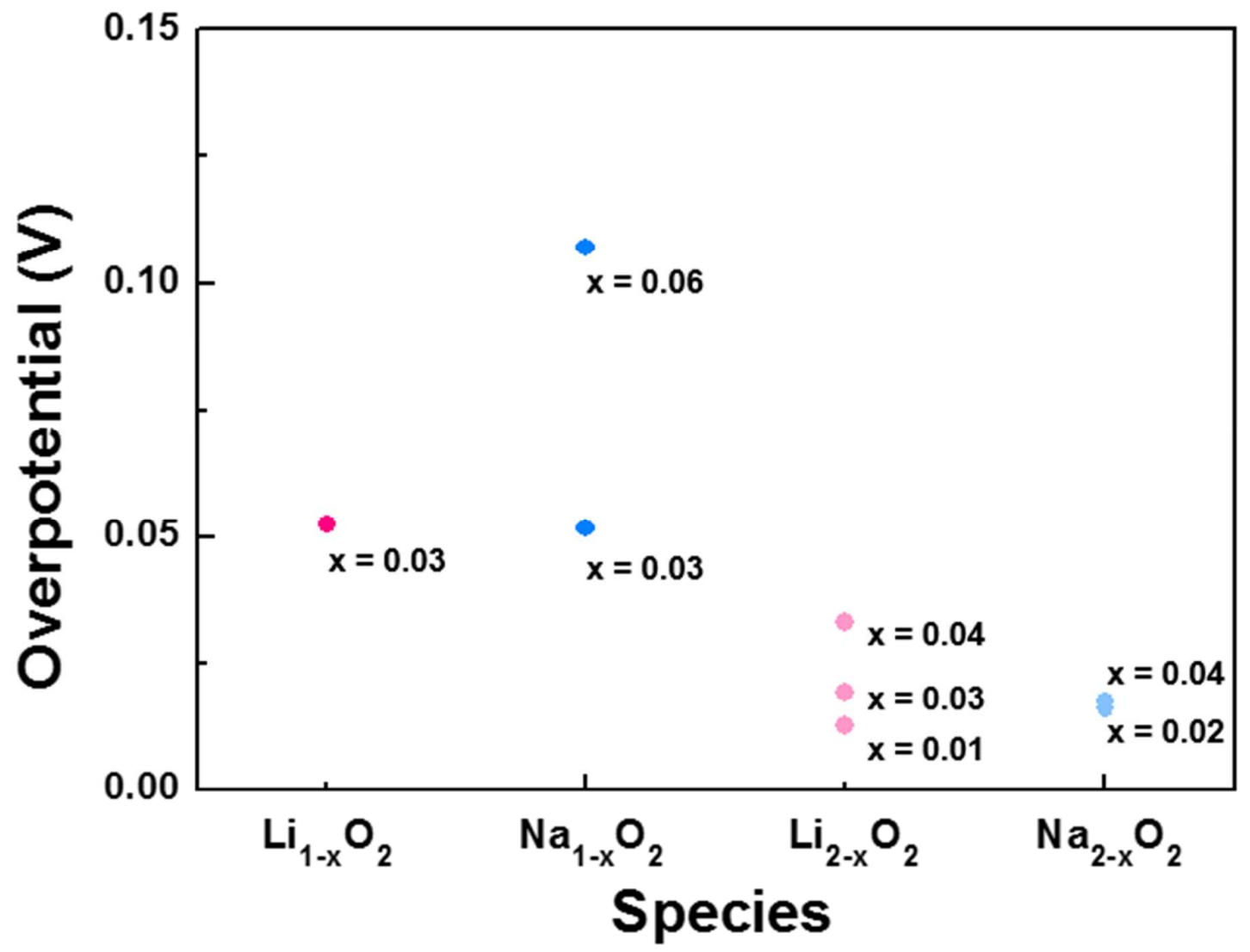

Figure S9. Required overpotential to form metal deficient phase of discharge product, $\mathrm{Li}_{1-\mathrm{x}} \mathrm{O}_{2}$, $\mathrm{Na}_{1-\mathrm{x}} \mathrm{O}_{2}, \mathrm{Li}_{2-\mathrm{x}} \mathrm{O}_{2}, \mathrm{Na}_{2-\mathrm{x}} \mathrm{O}_{2}, 0.01<\mathrm{x}<0.06$. 


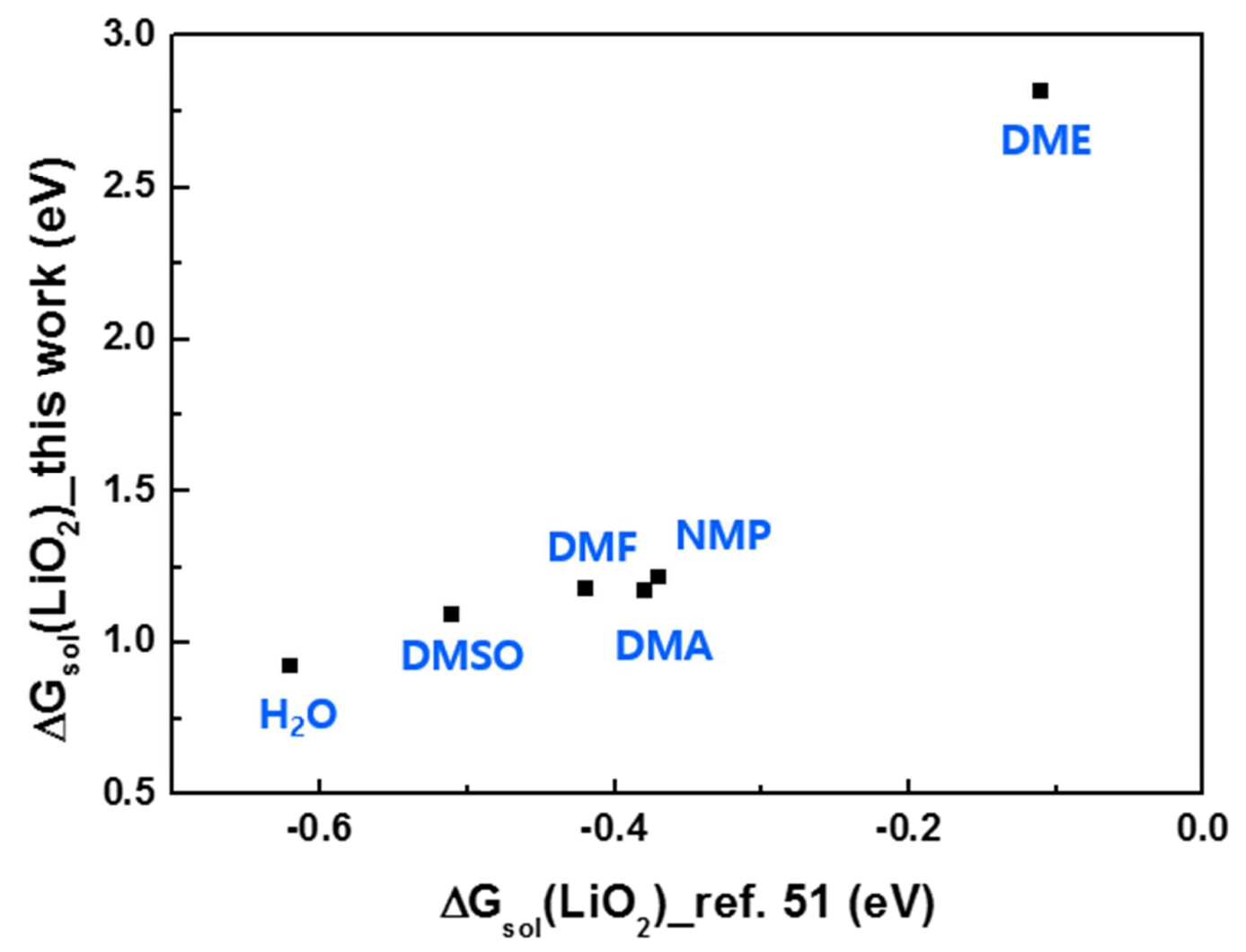

Figure S10. Relation between $\mathrm{LiO}_{2}$ ion dissolution energy (vs. intermediate product $\mathrm{LiO}_{2}$ at the surface) in ref. 51 and $\mathrm{LiO}_{2}$ ion dissolution energy (vs. bulk $\mathrm{LiO}_{2}$ ) in this study, calculated from interpolation of data in Figure 4(b), dielectric constants of $80.16\left(\mathrm{H}_{2} \mathrm{O}\right), 46.45$ (DMSO), 36.71 (DMF), 37.78 (DMA), 32.17 (NMP) and 7.2 (DME) were used. 